\title{
Contact tracing of a 15-year-old girl with smear- negative pulmonary tuberculosis in Tehran
}

N. Baghaie, ${ }^{7}$ S. Khalilzadeh, ${ }^{7}$ M.R. Bolursaz ${ }^{7}$ and N. Parsanejad ${ }^{7}$

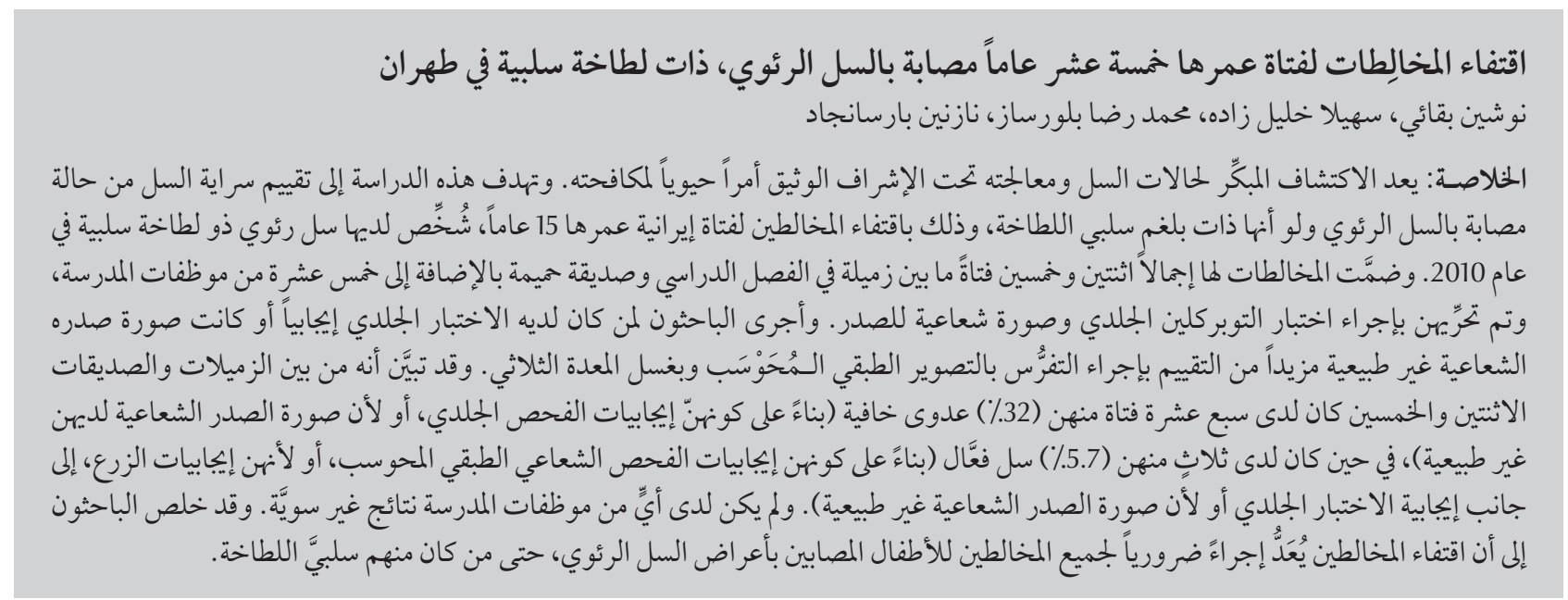

ABSTRACT Early detection of tuberculosis (TB) cases and supervised treatment are vital for control of the disease. To assess TB transmission from a sputum smear-negative pulmonary TB case, the contacts of a 15-year-old Iranian girl diagnosed with smear-negative TB in 2010 were traced. In all, 52 classmates and close friends and 15 school staff were screened by tuberculin skin test and chest X-ray. Those with a positive skin test or abnormal chest X-ray were further evaluated by chest spiral computed tomography $(\mathrm{CT})$ scan and triple gastric washing. All classmates and close friends were Iranian and female. Of the 52 girls, 17 (32\%) had latent infection (either positive skin test or abnormal chest X-ray) and 3 (5.7\%) had active TB (abnormal CT or positive culture additional to positive skin test or abnormal X-ray). None of the staff had abnormal findings. Contact tracing should be considered for contacts of all children with symptomatic pulmonary TB, even those who are smear-negative.

\section{Recherche des contacts d'une patiente âgée de 15 ans et atteinte d'une tuberculose pulmonaire à frottis négatif à Téhéran}

RÉSUMÉ Un dépistage précoce des cas de tuberculose et un traitement avec suivi régulier sont essentiels pour lutter contre la maladie. Pour évaluer la transmission de la tuberculose à partir d'un cas de tuberculose pulmonaire à frottis négatif, les contacts d'une patiente iranienne de 15 ans atteinte de tuberculose à frottis négatif en 2010 ont fait l'objet d'un suivi. En tout, 52 camarades de classes, amies proches et personnels de l'établissement scolaire ont été dépistés au moyen d'un test tuberculinique cutané et d'une radiographie des poumons. Une tomodensitométrie hélicoïdale thoracique et un triple lavage gastrique ont été réalisés chez les contacts présentant un test tuberculinique cutané positif ou une radiographie des poumons anormale. Toutes ses camarades de classe et amies proches étaient des Iraniennes. Sur les 52 filles, 17 d'entre elles (soit 32 \%) étaient porteuses d'une infection latente (test cutané positif ou radiographie des poumons anormale) et trois jeunes filles (soit 5,7 \%) étaient atteintes d'une tuberculose active (tomodensitométrie anormale ou culture positive, plus test cutané positif ou radiographie anormale). Aucun membre du personnel n'a présenté de résultats anormaux. La recherche des contacts doit être envisagée pour les personnes proches de tous les enfants atteints d'une tuberculose pulmonaire symptomatique, même dans les cas de frottis négatifs. 


\section{Introduction}

Tuberculosis (TB) is still one of the leading causes of mortality and morbidity. According to the World Health Organization (WHO), there are 9 million new cases of TB and 2 million deaths from the disease per year, $10 \%$ of which occur in children [1].

Since children are more asymptomatic or have weaker cough than adults, and usually have a smaller bacterial load, little attention has been paid to the transmission of TB from a child. Several TB outbreaks have been reported in schools but the index case was rarely a child $[2,3]$; the source of infection was usually adults. Early case detection and diagnosis of TB and supervised treatment are vital for control and reduction of TB and are part of the components of the Stop TB strategy [4]]. Thus, many studies have highlighted the importance of tracing the contacts of an adult smear-positive case $[5,6]$ but a few have investigated the contacts of a smearnegative child as an index case [7].

In 2010, a 15-year-old Iranian girl was admitted to the paediatric ward of Masih Daneshvari Hospital with fever, cough and haemoptysis. She had a history of contact with her grandmother who had been diagnosed with TB. Other members of her family were considered uninfected having been previously screened. High resolution computed tomography (HRCT) was suggestive of TB and Mycobacterium tuberculosis was isolated in culture of gastric aspirate. Following the diagnosis of $\mathrm{TB}$, she was started on treatment. This paper describes the screening undertaken of all the girls' classmates in the same grade at school and her close friends to determine if any were infected and reports the results of that screening.

\section{Methods}

All girls aged 15 years who were classmates or close friends of the index case (15-year-old Iranian girl diagnosed with active pulmonary $\mathrm{TB}$ ) and school staff were examined. As the school was not coeducational, only girls were screened.

Diagnosis of the TB was based on the criteria described below [8].

- 1. History of close contact

- 2. A positive purified protein derivative (PPD) test $(\geq 10 \mathrm{~mm})$

- 3. Radiographic findings

- 4. Presence of clinical symptoms

- 5. Positive pathology or bacteriology

The presence of at least 3 of these criteria are needed to establish a diagnosis of TB.

The index case had a history of contact with her grandmother living with them who was diagnosed with TB and she had died recently. She had also been symptomatic for 2 months; she had fever, cough and haemoptysis. HRCT showed interlobular nodules along with septal thickening (tree in bud) in the right lung. Gastric aspirate was negative, polymerase chain reaction (PCR) result of gastric juices was positive and 2 months later her culture was found positive too. Thus the index case fulfilled 3 criteria (close contact, symptoms and radiological findings compatible with TB) and diagnosis of TB was established for her and treatment initiated before receipt of her culture results.

All contacts were screened by a tuberculin skin (PPD) test and chest $\mathrm{X}$-ray initially and they were clinically examined. Tuberculin skin test was read by 2 paediatricians and an induration of $\geq 10 \mathrm{~mm}$ at 48 to 72 hours was considered positive $[9,10]$.

Contacts with negative PPD result were examined for the second time after 12 weeks to verify the PPD conversion (conversion is defined as changes in tuberculin skin test from $<10 \mathrm{~mm}$ in diameter to $>10 \mathrm{~mm}$ in diameter and $\mathrm{a}$ increase of $6 \mathrm{~mm}$ within a period of 2 years) [9]. Chest X-rays were taken in Masih Daneshvari Hospital and were reviewed by a radiologist and a paediatrician.
Any contact (children or staff) who had either a positive PPD or an abnormal chest X-ray were candidates for further evaluation. Triple gastric washing [11] (smear, culture, PCR) and a spiral CT scan [12] were carried out for them.

Depending on the results of the tuberculin skin test, chest radiograph, chest spiral CT scan and gastric aspirate smear and culture for M. tuberculosis, contacts were classified as uninfected, infected or active TB. Contacts who had either a positive PPD $(\geq 10 \mathrm{~mm})$ or a suspicious chest X-ray were considered infected. Those contacts who had a positive gastric aspirate or a positive chest spiral CT along with a positive PPD or chest X-ray were identified as an active TB case.

Contacts with latent TB infection were prescribed isoniazid prophylaxis for 6 months, cases with active TB were started on the recommended 4-drug regimen (isoniazid, rifampin, pyrazinamide, ethambutol) [13].

This study was approved by the Ethics Committee of Masih Daneshvari Hospital. All participants were informed about the study and gave their written consent to participate.

\section{Results}

In all, 52 classmates/close friends and 15 staff were screened. The classmates/ close friends were 15 years old, Iranian, female and had received the BCG vaccination.

All contacts were asymptomatic but of the 52 girl contacts, 15 had a positive PPD and 1 was observed to have tuberculin conversion; her PPD converted from $5 \mathrm{~mm}$ to $15 \mathrm{~mm}$ after 12 weeks. Chest X-ray was abnormal in 6 contacts; hilar adenopathy was the commonest finding.

Of the 52 girl contacts, 20 were selected for further evaluation. Of these, $3(5.8 \%)$ showed positive results and according to the criteria listed earlier 
were diagnosed as active TB. The first case had a positive PPD of $35 \mathrm{~mm}$ and positive gastric aspirate culture. The second had a PPD of $25 \mathrm{~mm}$ and a calcified nodule in spiral CT. The third had a PPD of $18 \mathrm{~mm}$ and unilateral adenopathy in spiral CT. All 3 cases had a history of close contact with the index case and thus fulfilled the diagnostic criteria. All were started on the recommended 4-drug regimen.

The other 17 contacts (32\%) were considered to be infected because of either a positive tuberculin skin test or abnormal chest X-ray and they were prescribed isoniazid prophylaxis for 6 months.

All the school staff examined had unremarkable results for the screening tests and none therefore was considered to have latent or active TB.

\section{Discussion}

Previous studies have proposed that the risk caused by smear-negative patients cannot be ruled out and they all suggested that the contacts of smear-negative patients should be examined [14-16]. This study is one of the very few [7] to document the transmission of $\mathrm{TB}$ from a smear-negative child.

Our index case had a negative sputum culture but she was symptomatic for a few months and she continued to attend school while symptomatic. Thus screening was scheduled for all her close contacts in the same class and school staff.

Of the 52 students, 17 (32\%) were considered to have latent infection. This is lower than a study in England where the infection rate in the class of the smear-negative index case was $79 \%$ and was $35 \%$ for other students in the school [7]. Furthermore, 3 (5.7\%) cases of active TB were identified. This is not a very high rate when compared to a study conducted in a junior school in south-eastern England, where TB was diagnosed in $9 \%$ of contacts [2]. In several retrospective contact tracing studies, $90 \%$ or more of TB cases were identified within the first 12 months after identification of the index case $[17,18]$. We were not able to follow up the patients for this length of time because of lack of cooperation of the TB cases and lack of a screening programme in our country, which may explain why our result is lower than the other studies.

We believe that with the high rate of infection among the classmates, the index child was the likely source of the infection. In addition, exposure to adult active TB in the school was ruled out. In this situation, DNA fingerprinting could be useful for confirming the index case as the source of infection. Because of financial constraints, fingerprinting to identify the index case was not done, which is another limitation of our study.

\section{Conclusion}

Our study suggests that children with TB who may even have a negative smear can transmit infection to others and therefore should be considered infectious until proven otherwise. Contact tracing should be considered for contacts of children with symptomatic pulmonary tuberculosis.

\section{References}

1. Global Tuberculosis control: surveillance planning, financing. Geneva, World Health Organization, 2006.

2. Howard J et al. Outbreak of tuberculosis in south-eastern England. Eurosurveillance, 2007;12(6).

3. Philip L, Carlile J, Smith D. Epidemiology of tuberculosis outbreak in a rural Missouri High school. Pediatrics, 2004, 113:e514-e519.

4. World Health Organization. The Stop TB Strategy. Vision, goal, objectives and targets (http://www.who.int/tb/strategy/stop_tb_strategy/en/index.html, accessed 31 January 2012).

5. Beyers $\mathrm{N}$ et al. A prospective evaluation of children under the age of 5 years living in the same household as adults with recently diagnosed pulmonary Tuberculosis. International Journal of Tuberculosis and Lung Disease, 1997, 1:38-43.

6. Donland PR. Preventing tuberculosis in childhood. Indian Journal of Pediatrics, 2000, 67:383-385.

7. Paranjothy $\mathrm{S}$ et al. Extensive transmission of Mycobacterium tuberculosis from 9 year old child with pulmonary tuberculosis and negative sputum smear. BMJ (Clinical Research Ed.), 2008, 337:a1184.

8. Baghaie $\mathrm{N}$ et al. Diagnostic scoring system for pediatric tuberculosis. Journal of the Medical Council of IRI, 1999, 16:287-292 [In Farsi].
9. Seth V, Kabara SK, Seth R. Essentials of tuberculosis in children, 3rd ed. New Delhi, Jaypee Brothers, 2006:323-339.

10. Khalilzadeh $\mathrm{S}$ et al. Screening of tuberculosis in symptomatic close contact children. Tanaffos, 2003, 2:51-56.

11. Schaaf HS et al. Evaluation of young children in household contact with adult multidrug resistant pulmonary tuberculosis cases. Pediatric Infectious Disease Journal, 1999, 18:494-500.

12. Baghaie $\mathrm{N}$ et al. Diagnostic value of lung $\mathrm{CT}$ scan in childhood tuberculosis. Tanaffos, 2005, 4:57-62.

13. Seth V, Kabara SK, Seth R. Essentials of tuberculosis in children, 3rd ed. New Delhi, Jaypee Brothers, 2006:435-447.

14. Behr MA et al. Transmission of Mycobacterium tuberculosis from patients smear-negative for acid-fast bacilli. Lancet, 1999, 353:444-449.

15. Tostmann A et al. Tuberculosis transmission by patients with smear negative pulmonary tuberculosis in a large cohort in The Netherlands. Clinical Infectious Diseases, 2008, 47:1135-1142.

16. Hernández-Garduño E et al. Transmission of tuberculosis from smear negative patients: a molecular epidemiology study. Thorax, 2004, 59:286-290.

17. Ormerod LP. Rules of tuberculosis contact tracing : Blackburn 1962-1990. Respiratory Medicine, 1993, 87:127-131.

18. Teale C, Cundall DB, Pearson SB. Time of development of tuberculosis in contacts. Respiratory Medicine, 1991, 85:475-477. 